\title{
A new type of antiferrodistortive structure in a double
}

\section{perovskite with $\mathrm{Pb}$}

Javier Blasco ${ }^{*}$, Sara Lafuerza, Joaquín García, Gloria Subías, Mª Concepción Sánchez, Vera Cuartero and Jolanta Stankiewicz.

Instituto de Ciencia de Materiales de Aragón, Departamento de Física de la Materia Condensada, CSIC-Universidad de Zaragoza. C/ Pedro Cerbuna 12, 50009 Zaragoza, Spain.

\section{Corresponding author:}

Javier Blasco

Instituto de Ciencia de Materiales de Aragón

CSIC-Universidad de Zaragoza

EMAIL ADDRESS: jbc@unizar.es

KEYWORDS: Multiferroic, phase transition, double perovskite, antiferroelectric, ferrimagnetism. 


\begin{abstract}
We find a new structural transition in $\mathrm{Pb}_{2} \mathrm{MnReO}_{6}$ at $410 \mathrm{~K}$. Above this temperature, $\mathrm{Pb}_{2} \mathrm{MnReO}_{6}$ is cubic with disordered and dynamic atomic displacements manifested in the large thermal parameters

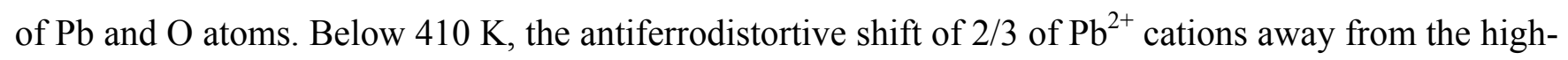
symmetry cubic site produces a new type of monoclinic cell. The unit cell expands at the transition and the heat capacity shows a peak with thermal hysteresis. These features agree with a first order transition. The entropy content of the transition is quite low indicating that the structural disorder has not been completely removed in the low temperature phase.
\end{abstract}

The monoclinic phase of $\mathrm{Pb}_{2} \mathrm{MnReO}_{6}$ shows thermally activated conductivity which does not vary when an external magnetic field is applied. A change in the slope of the resistivity curve, observed at the structural phase transition temperature, is related to a slight difference in the activation energy between both phases. It suggests that the condensation of the distortions likely affects the conduction mechanism. The isothermal magnetization measurements reveal the presence of ferromagnetic contributions below $85 \mathrm{~K}$. The ac magnetic susceptibility shows a dynamic peak at $50 \mathrm{~K}$ and, in addition, zero-field-cooled and field-cooled magnetization curves diverge strongly below $80 \mathrm{~K}$. These features might be signature of magnetic inhomogeneity. Magnetic loops, obtained at $5 \mathrm{~K}$, do not show saturation in fields up to $9 \mathrm{~T}$. Furthermore, the measured coercivity increases sharply at low temperature indicating an abrupt change in the magnetic anisotropy. We show that all these magnetic properties point out to a ferrimagnetic ordering of $\mathrm{Mn}$ and Re atoms in an intermediate valence state. 


\section{Introduction.}

Materials which integrate magnetic and electric orderings are of great interest since they show not only intriguing physical properties ${ }^{1}$ but they are also attractive for technological applications. ${ }^{2-3}$ The most useful attribute is the coupling between electric and magnetic properties which gives rise to the magnetoelectric effect. This effect has been found recently in several oxides with perovskite-like structures. ${ }^{1-6}$ The perovskite oxide, $\mathrm{ABO}_{3}$, has space group $P m \overline{3} m$ with a 12 -fold coordinated large cation (A) and an octahedrally coordinated smaller cation (B). The solid solution of type $\mathrm{AB}_{1-\mathrm{x}} \mathrm{B}_{\mathrm{x}} \mathrm{O}_{3}$ is commonly found for these compounds. However, if the B and B' cations differ in oxidation state or in size or in both, then the B-site cations are very likely to order in a rock-salt pattern. Consequently, the new phase is described by the formula $\mathrm{A}_{2} \mathrm{BB}^{\prime} \mathrm{O}_{6}$ for $\mathrm{x}=0.5$ and is denoted as double perovskite (also elpasolite). ${ }^{7-9}$ Ferro- and antiferromagnetic B-B' couplings are usually observed in these compounds and in some cases, even with a non-magnetic B element. ${ }^{10}$

A current research activity in the field of double perovskites is motivated by the finding that the stereochemical activity of the $6 \mathrm{~s}^{2}$ lone pair could favour the coupling between ferroelectricity and magnetism. Such mechanism might take place in $\mathrm{Bi}^{3+}$ and $\mathrm{Pb}^{2+}$ double perovskites with magnetic interactions between B and B' cations. The above strategy has mainly been used for Bi-based compounds. ${ }^{6,11}$ However, $\mathrm{Pb}_{2} \mathrm{BB}^{\prime} \mathrm{O}_{6}$ compounds have been known for a long time. ${ }^{12-14}$ In these compounds, the $\mathrm{Pb}$ usually adopts distorted environments which arises from displacements of $\mathrm{Pb}$ ions out from the centre of the oxygen coordination polyhedra. The displacements may occur along either the [100] cubic direction as reported for $\mathrm{Pb}_{2} \mathrm{CoWO}_{6}$ and $\mathrm{Pb}_{2} \mathrm{MnWO}_{6}$ double perovskites ${ }^{15,16}$ or the [111] cubic direction as in the $\mathrm{Pb}_{2} \mathrm{ScSbO}_{6}$ compound. ${ }^{17}$ In most cases, reported studies focused on electrical properties of samples with non-magnetic B-cations. Here, we report results of a study on $\mathrm{Pb}_{2} \mathrm{MnReO}_{6}$, where both $\mathrm{Mn}$ and Re have unpaired $d$ electrons and the superexchange rules predict a ferrimagnetic ground state. Indeed, a magnetic transition was found below $100 \mathrm{~K}$ but, surprisingly, no distortive displacements of the $\mathrm{Pb}$-cation have been reported in an earlier work. ${ }^{18}$ The crystal structure at room temperature has been refined as a monoclinic double perovskite (space group $P 2_{l} / n$ ) arising from 
cooperative tilts of the $\mathrm{BO}_{6}$ octahedra. Apparently, the stereochemical activity of the $6 \mathrm{~s}^{2}$ lone pair in $\mathrm{Pb}^{2+}$ has been quenched for this compound.

In the course of our search for double-perovskites with enhanced multiferroic properties, we have depicted the para-antiferroelectric phase transition in $\mathrm{Pb}_{2} \mathrm{MnWO}_{6}$ which occurs at $423 \mathrm{~K} .^{16,19}$ This compound also undergoes a structural phase transition at the same temperature. ${ }^{16}$ The high temperature phase is cubic and transforms into an orthorhombic structure with collinear antiferroelectric displacements of $\mathrm{Pb}^{2+}$ cations. These atoms have a distorted first coordination shell. EXAFS spectroscopy reveals a similar local disorder around $\mathrm{Pb}$ atoms for both cubic and orthorhombic phases. ${ }^{19}$ Hence, dynamic disordered distortions exist in the cubic phase, which freeze in an ordered pattern below $423 \mathrm{~K}$, and the transition can be classified as an order-disorder type. We expected that the replacement of W by Re would affect the magnetic properties of the system but we did not suspect that this substitution will deeply modify the local structure for $\mathrm{Pb}^{2+}$.

A likely coupling between magnetism and crystal structure motivated us to carry out a careful characterization of the crystallographic, electrical and magnetic properties of $\mathrm{Pb}_{2} \mathrm{MnReO}_{6}$. It is noteworthy that the stoichiometry of this compound is highly dependent on the synthetic conditions. The perovskite structure can only be obtained at relatively low temperature. At higher temperatures, a competitive pyrochlore phase is formed. ${ }^{18}$ We successfully synthesized the perovskite phase with a full Mn-Re ordering at the octahedral sites for the first time. We found that this system undergoes a structural phase transition above room temperature, similarly to $\mathrm{Pb}_{2} \mathrm{MnWO}_{6}$. Our results show a new type of monoclinic cell at room temperature. This monoclinic structure actually shows antiferroelectric movements of some $\mathrm{Pb}$ atoms in a way not previously observed in any other double perovskite. Electrical and magnetic properties reasonably agree with the ones previously reported. ${ }^{18}$ Slight differences might be ascribed to the different synthetic route used in the present work that allowed us to prepare a sample free of antisite defects. The reported structural phase transition is accompanied by an anomaly in the resistivity curve. A ferrimagnetic ordering of $\mathrm{Mn}$ and Re atoms in a mixed valence state is also observed below $85 \mathrm{~K}$. 


\section{Experimental Section.}

$\mathrm{Pb}_{2} \mathrm{MnReO}_{6}$ was prepared by solid state chemistry reaction. Stoichiometric amounts of $\mathrm{PbO}, \mathrm{MnCO}_{3}$ and $\mathrm{ReO}_{3}$ were mixed, ground, pressed into pellets and heated in an $\mathrm{Ar}$ stream at $710^{\circ} \mathrm{C}$ for $2 \mathrm{~h}$ twice with an intermediate ground. Previous synthesis was carried out at $550^{\circ} \mathrm{C}$ for $12 \mathrm{~h}$ and the starting precursors were $\mathrm{Re}_{2} \mathrm{O}_{7}, \mathrm{Mn}_{2} \mathrm{O}_{3}$ and $\mathrm{PbO} .{ }^{18}$ We have observed that the perovskite phase is formed faster (kinetically controlled product) than the pyrochlore phase (thermodynamically controlled product) and the direct synthesis at higher temperature with shorter reaction times also yields a pure perovskite phase. The temperature limit is around $725^{\circ} \mathrm{C}$. Above this limit, significant amount of pyrochlore phase is formed while below this temperature the reaction time is also important because long reaction times favour the appearing and growing of the pyrochlore phase. The use of $\mathrm{ReO}_{6}$ and $\mathrm{MnCO}_{3}$ precursors, with the appropriate cationic valences, also seems to favour the kinetic control of the chemical reaction. The increase in the temperature reaction improves the Re-Mn ordering as occurs in related double perovskites. $^{20}$

The resulting pellet was black with a high electrical resistivity. The sample was characterized by $\mathrm{x}-$ ray powder diffraction using a Rigaku D-Max system. Step-scanned patterns were measured between $10^{\circ}$ and $130^{\circ}$ (in steps of $0.03^{\circ}$ ) at room temperature. The $\mathrm{x}$-ray system was working at $40 \mathrm{kV}$ and 100 $\mathrm{mA}$ with a counting rate of 4 sec.step ${ }^{-1}$. We used a graphite monochromator to select the $\mathrm{Cu} \mathrm{K}_{\alpha}$ radiation. The medium and low temperature chamber manufactured by Rigaku was coupled to the goniometer to measure patterns between 123 and $523 \mathrm{~K}$. In this case, the angle range was between $10^{\circ}$ and $120^{\circ}$ with the same step. The crystal structures were refined by the Rietveld method using the Fullprof package program. ${ }^{21}$

Differential scanning calorimetry (DSC) was measured using a DSC Q-20 from TA-Instruments with samples sealed in aluminium pans under a nitrogen atmosphere. The scanning rate was $10 \mathrm{Kmin}^{-1}$. Magnetic measurements were performed between 5 and $300 \mathrm{~K}$ in a commercial Quantum Design superconducting quantum interference device (SQUID) magnetometer. Resistivity measurements were carried out using the standard six-probe AC method in the temperature range between 80 and $500 \mathrm{~K}$. An 
electromagnet, which provides magnetic fields up to $1 \mathrm{~T}$, was used to test the magnetoresistance properties.

\section{Results and discussion}

3.1. Structure. Our crystallographic study reveals that $\mathrm{Pb}_{2} \mathrm{MnReO}_{6}$ undergoes a structural phase transition upon heating above room temperature, which resembles the one observed in the $\mathrm{Pb}_{2} \mathrm{MnWO}_{6}$ system. ${ }^{16}$ Figure 1 shows the x-ray diffraction pattern collected at $523 \mathrm{~K}$. This measurement confirmed that our sample was almost single phase with only a tiny impurity $(0.9 \%$ in weight $)$ identified as a pyrochlore phase with a similar chemical composition. The secondary phase was included in the refinement using the structural data reported previously. ${ }^{18}$ At $523 \mathrm{~K}, \mathrm{~Pb}_{2} \mathrm{MnReO}_{6}$ adopts the ordered cubic perovskite structure with the space group $F m \overline{3} m$. The Rietveld refinement confirms a full Mn-Re ordering without significant presence of antisite defects (i.e. Mn-Re substitution disorder). This is an important difference with the previous study on $\mathrm{Pb}_{2} \mathrm{MnReO}_{6}$ where $15 \%$ of antisite disorder was found. ${ }^{18}$ The difference might come from the higher synthesis temperature used in the present study as it is indicated in the experimental section. The structural parameters derived from the refinement of this pattern are summarized in tables 1 and 2. Unusual large temperature factors associated with the $\mathrm{Pb}$ and $\mathrm{O}$ sites indicate the presence of disordered atomic displacements in the cubic phase. Similar results have been reported in the cubic phases of other Pb-based double perovskites and they have been ascribed to the dynamic distortions induced by the steroactive electron lone-pair of $\mathrm{Pb}^{2+} \cdot{ }^{14-17}$

Table 3 reports selected interatomic distances obtained from the structural refinement. Regarding the $\mathrm{Pb}-\mathrm{O}$ polyhedron, the experimental value reported in table 3 agrees with the theoretical estimation of $2.87 \AA$ for $\mathrm{Pb}^{2+}-\mathrm{O}$ distance. The distances obtained for the cubic phase reveal the presence of regular $\mathrm{MnO}_{6}$ and $\mathrm{ReO}_{6}$ octahedra. Assuming the tabulated ionic radia, ${ }^{22}$ the calculated values for $\mathrm{Mn}^{2+}-\mathrm{O}$, $\mathrm{Mn}^{3+}-\mathrm{O}, \mathrm{Re}^{5+}-\mathrm{O}$ and $\mathrm{Re}^{6+}-\mathrm{O}$ bond lengths should be $2.23,2.085,1.98$ and $1.95 \AA$, respectively. The comparison to the experimental values of table 3 suggests an intermediate valence for Re and a valence close to +3 for $\mathrm{Mn}$ ion. However, experimental $\mathrm{Mn}^{2+}-\mathrm{O}$ distances found in Mn-based double perovskites $^{16,23}$ range between 2.12 and $2.14 \AA$. Therefore, we can assume an intermediate valence for 
$\mathrm{Mn}$ in the $\mathrm{Pb}_{2} \mathrm{MnReO}_{6}$ as well. In this way, $\mathrm{Mn}^{2.5+}$ and $\mathrm{Re}^{5.5+}$ seem to be the most plausible intermediate valence states for the $\mathrm{Pb}_{2} \mathrm{MnReO}_{6}$ double-perovskite.

Upon decreasing the temperature, two changes occur in the x-ray diffraction patterns below $390 \mathrm{~K}$, as can be observed in Fig. 2. One is the peak splitting that develops in many of the strong diffraction lines. The second change is the appearing of a number of new superstructure peaks as is shown in the inset of Fig.2. The splitting of the peaks can be accounted for by the monoclinic cell reported in Ref. 18 (space group $P{ }_{1} / n$ ). This cell forms from the cooperative tilts of $\mathrm{MnO}_{6}$ and $\mathrm{ReO}_{6}$ octahedra, following the system tilt denoted as $\mathrm{a}^{-} \mathrm{a}^{-} \mathrm{c}^{+}$in the Glazer's terminology. ${ }^{24}$ However, an exhaustive inspection of the $\mathrm{x}-$ ray patterns casts doubts on such interpretation. In addition, a monoclinic cell with space group $I 2 / m$ also fits the main peaks of the pattern. The latter monoclinic cell corresponds to the $\mathrm{a}^{0} \mathrm{~b}^{-} \mathrm{b}^{-}$tilt system. ${ }^{24}$ In any case, both these models fail to reproduce the intensity of the most important new superstructure peaks indicating that not only cooperative octahedra tilts but also atomic shifts are responsible for their presence. Details of the refinements using $I 2 / m$ and $P 2_{1} / n$ space groups are available in the additional supporting information accompanying this article. The indexation of the main superstructure peaks using the high temperature structure (inset of Fig. 2) suggests the need to treble a monoclinic axis (a face diagonal of the high temperature phase). In order to test it, we have verified by means of a Lebail refinement ${ }^{25}$ that these new lines can be indexed after trebling the b-axis, i.e. within a monoclinic cell with lattice vectors $(1 / 2,1 / 2,0),(-3 / 2,3 / 2,0)$ and $(0,0,1)$ with respect to the parent structure. Only four very weak reflections that we will discuss later on were not indexed (two of them are underlined in the inset of Fig. 2).

In order to explore the possible atomic displacements able to give the mentioned triple monoclinic cell, we have used the ISODISPLACE tool. ${ }^{26}$ This software allows us to explore structural phase transitions by generating atomic displacement patterns corresponding to irreducible representations of the parent space-group symmetry. Accordingly, we have used the cubic double perovskite structure (high temperature phase) as the parent compound and we have searched over arbitrary k-points of the first Brillouin zone in order to obtain the mentioned 'triple' monoclinic cell or related ones. A detailed 
inspection of the active modes for the different solutions revealed that the main superstructure peaks arise from the condensation of a $\Sigma_{2}$ mode at the $(2 / 3,2 / 3,0) \mathrm{k}$-point. Three solutions were retained as the most plausible candidates and they are summarized in table 4. Among these solutions, the C-centred lattice implies the least amount of active modes and, therefore, fewer parameters to be refined. In order to test it, we have used the AMPLIMODES program ${ }^{27}$ from the Bilbao Crystallographic Server to obtain an input file for Fullprof. The structural analysis consists of decomposing the symmetry-breaking distortion, present in the transition from $F m \overline{3} m$ to $C 2 / m$, into contributions from various symmetryadapted modes. The amplitude refinement of the basis modes reaches convergence easily and gives rise to an accurate fit as the one shown in the Fig. 2. Details of the model and the final atom positions are summarized in tables 1 and 2, respectively. The refined global amplitudes are indicated in table 4 . We have performed similar refinements using the other two solutions of table 4 and we obtained similar good fits if only the same modes present in the $C 2 / m$ cell were used. Additional modes, specific to $P 2{ }_{1} / c$ or $P 2 / c$ cells, have negligible contribution in the refinement process. It strongly suggests that the $C 2 / m$ solution is the simplest model accounting for the x-ray pattern. The only exception are four very weak peaks indexed in the frame of the parent compound as $(4 / 3,4 / 3,5 / 2),(2 / 3,2 / 3,7 / 2),(2 / 3,2 / 3,9 / 2)$ and $(14 / 3,14 / 3,3 / 2)$. Two of them are marked in the inset of fig. 2 for the sake of comparison. These lines point to the doubling of another axis. The symmetry analysis yields as a possible solution a primitive monoclinic cell whose lattice vectors respect to the parent compound are $(-1 / 2,-1 / 2,0),(0,0,2)$ and $(-1$, 2, 0). We have checked that a Lebail refinement using the highest possible symmetry $\left(P 2_{1} / c\right.$ space group) can account for all diffraction peaks. However, it contains 90 amplitudes of basis modes whose refinement is out of the accuracy of our conventional x-ray powder diffraction patterns. Therefore, we have retained the $C 2 / m$ solution as the best structural approximation for $\mathrm{Pb}_{2} \mathrm{MnReO}_{6}$.

The crystal structure of $\mathrm{Pb}_{2} \mathrm{MnReO}_{6}$ at room temperature is a new type of monoclinic structure that has not been reported, up to the best of our knowledge, in the field of double perovskites. In this structure, the crystallographic site of $\mathrm{Pb}, \mathrm{Mn}$, and Re atoms is split into two non-equivalent sites with a ratio of 2 to 1 . The oxygen has 8 non-equivalent sites in the $C 2 / m$ cell. Such crystal structure can be 
understood as arising from the conventional cubic structure of a double perovskite by the combined condensation of two modes of atomic displacement, $\Gamma_{4}{ }^{+}$and $\Sigma_{2}$. The first one is associated to the antiphase tilts of $\mathrm{BO}_{6}$ octahedra about the $y$-direction in the monoclinic cell (or about the unique axis). Figure 3 shows a projection of the crystal structure onto the $a c$-plane emphasizing these tilts. The mode $\Sigma_{2}$ is associated to atomic shifts of the atoms located at some specific Wyckoff positions. The main shift corresponds to the $\mathrm{Pb}$ atoms placed at the $8 j$ positions (denoted as $\mathrm{Pb} 2$ in the table 2). This mode does not affect the $\mathrm{Pb}$ situated at the $4 h$ site $(\mathrm{Pb} 1)$. The $\mathrm{Pb} 2$ atoms are shifted by $\sim 0.177 \AA$ away from the ideal cubic position along the monoclinic $z$-direction which correspond to the $\left[\begin{array}{lll}1 & 1 & 0\end{array}\right]$ direction in the high temperature phase. The shift is in the opposite directions $(+z$ and $-z)$ for successive rows of $\mathrm{Pb} 2$ atoms giving rise to the antiferroelectric configuration indicated by arrows in Fig. 3. Only two thirds of the $\mathrm{Pb}$ atoms are shifted in opposite directions whereas the other third $(\mathrm{Pb} 1)$ remains fixed at $z=1 / 2$.

The $\Sigma_{2}$ mode also affects $\mathrm{Mn}$ atoms at one of the $\mathrm{Mn}$ sites although in lower proportion. Its contribution to the Re atoms is negligible. The remaining modes can be considered as secondary but they also play a role. For instance, the $\Sigma_{1}$ mode is associated with an antiphase displacement between $\mathrm{Pb} 1$ and $\mathrm{Pb} 2$ atoms along the $y$-direction. $\Gamma_{5}^{+}$, in addition to $\Sigma_{2}$, leads to oxygen displacements which distort the octahedral environment around $\mathrm{Mn}$ and Re atoms (see Fig. 3).

Regarding the atom bonds, there are significant differences between both crystallographic phases. $\mathrm{Pb}$ has a 12-fold coordination in the high temperature phase. Similar coordination with almost the same average $\mathrm{Pb}-\mathrm{O}$ distance remains for the $\mathrm{Pb} 1$ in the monoclinic phase (see table 3) although the local environment is more distorted. However, the atomic displacement of $\mathrm{Pb} 2$ atoms reduces their effective coordination to 9 and shortens the average $\mathrm{Pb} 2-\mathrm{O}$ bond length. In the case of Mn atoms, a distortion in the octahedral coordination is observed in the monoclinic phase with a slight difference in the size of $\mathrm{MnO}_{6}$ octahedra. Similar features are observed for the $\mathrm{ReO}_{6}$ octahedra but, in this case, the distortion is larger and both $\mathrm{ReO}_{6}$ octahedral expand through the transition to the monoclinic phase. Accordingly, the Re-O bond length increases towards the expected values for $\mathrm{Re}^{5+}-\mathrm{O}$. 
The temperature dependence of the lattice parameters upon decreasing the temperature from $523 \mathrm{~K}$ down to $123 \mathrm{~K}$ is displayed in Fig. 4. The structural transition is observed at $410 \mathrm{~K}$. Above this temperature, the cubic lattice parameter follows the typical thermal expansion on warming. Below 410 $\mathrm{K}$, only the monoclinic $c$-axis contracts monotonically on cooling. The other two monoclinic axes show anomalous behaviour: the $b$-axis abruptly shrinks for and the $a$-axis expands strongly. These changes produce an abrupt increase of $0.23 \AA^{3}$ in the unit cell volume per formula unit at the transition on cooling. Similar results were found for the structural transition of $\mathrm{Pb}_{2} \mathrm{MnWO}_{6} \cdot{ }^{16}$ Away from the transition region, the thermal expansion is positive in the whole temperature range studied although with different slopes for each phase. The volume expansion coefficient is $1.56 \times 10^{-3} \AA^{3} \times \mathrm{K}^{-1}$ for the monoclinic phase (between 123 and $373 \mathrm{~K}$ ) but it reaches a value of $5.44 \times 10^{-3} \AA^{3} \times \mathrm{K}^{-1}$ in the cubic phase between 423 and $523 \mathrm{~K}$. The evolution of the volume per unit formula versus temperature is included in the additional supporting information.

3.2. Heat capacity. The phase transition was also characterized by DSC measurements whose results are displayed in Fig. 4. Heat capacity shows a rounded peak at approximately $413 \mathrm{~K}$, in agreement with the change observed in the crystal structure. We have measured heating and cooling scans at the speed of $10 \mathrm{~K} \mathrm{~min}^{-1}$. A clear shift of $6 \mathrm{~K}$ is observed which agrees with a first order transition. The phasetransition enthalpy, $\Delta H=\int \Delta C \mathrm{p}(T) \mathrm{d} T$, is $768 \pm 58 \mathrm{~J} \mathrm{~mol}^{-1}$. The total change in the entropy is $\Delta S=1.86$ $\pm 0.15 \mathrm{Jmol}^{-1} \mathrm{~K}^{-1}$. This value is approximately equal to $0.22 R, R$ being the gas constant. The obtained value of entropy is smaller than the ones found for other double perovskites, which are usually close ${ }^{28}$ to $R \ln 4$. In addition, it is only one third of the corresponding value for the transition in $\mathrm{Pb}_{2} \mathrm{MnWO}_{6}{ }^{16}$ However, it is quite close to the entropy change observed in the structural transition of $\mathrm{Pb}_{2} \mathrm{MgTeO}_{6}$ $(\sim 0.3 R)$ which was ascribed to the presence of structural disorder in the low temperature phase. ${ }^{29}$ In our case, large temperature factor has been refined for $\mathrm{Pb} 1$ at room temperature (see table 3) suggesting that dynamic disorder is still present for this crystallographic site.

3.3. Electrical properties. The electrical resistivity was measured from 500 down to $80 \mathrm{~K}$. Below this temperature, the resistivity was to high to be measured reliably. The $\mathrm{Pb}_{2} \mathrm{MnReO}_{6}$ system is 
semiconducting in the temperature range studied (Fig. 5). It shows an anomaly in the curve slope at the structural transition temperature which is clearly seen in the plot of Ln $\rho$ vs. 1/T exhibited in the inset of Fig. 5. Above and below the phase transition temperature, the Arrhenius law is obeyed. The change in the slope is then ascribed to an increase of the activation energy at the transition which can be related to the enlargement of the energy gap in the low temperature phase. Above the transition, the linear fit gives the activation energy of $0.135 \mathrm{eV}$ whereas below the transition, the linear fit works down to $150 \mathrm{~K}$ (see additional information) and the activation energy is $0.19 \mathrm{eV}$. Overall, the later value agrees with the ones reported in Ref. 18; the slight difference may be ascribed to the different degree of antisite disorder. We have repeated the electrical measurement under an external field of $1 \mathrm{~T}$. No differences have been found between both curves indicating a negligible magnetoresistance in these conditions.

Attempts of characterizing the dielectric permittivity at the structural transition were unsuccessful due to the high electronic conductivity of the sample. The electrical resistivity measured at 300 and $400 \mathrm{~K}$ was $\sim 2$ and $\sim 0.27 \Omega . c m$, respectively. Therefore, possible anomalies in the dielectric constant were masked by the high conductivity of the material. Consequently, an ordering of electric dipoles at the transition cannot be excluded.

3.4. Magnetic properties. The temperature dependence of the dc magnetization was measured using zero-field-cooling (ZFC) and field-cooling (FC) conditions at two applied magnetic fields as can be seen in the Fig. 6. At $500 \mathrm{Oe}$, the $\mathrm{ZFC}$ curve shows a peak at $40 \mathrm{~K}$ and a divergence between $\mathrm{FC}$ and ZFC curves begins at $77 \mathrm{~K}$. These features strongly depend on the magnetic field strength. At $5 \mathrm{kOe}$, the maximum in the ZFC curve is observed at $20 \mathrm{~K}$ and the magnetic irreversibility starts at $47 \mathrm{~K}$. In both cases, the FC curves behave as expected for a ferri- or ferromagnetic transition.

The ac magnetic susceptibility $\left(\chi_{\mathrm{ac}}\right)$ shows a broad maximum at $51 \mathrm{~K}$ whose intensity depends on the frequency of the alternating field (see inset of Fig. 6). There is no significant shift of the magnetic peak in the frequency range studied $(17-999 \mathrm{~Hz})$. Both properties, magnetic irreversibility in dc magnetization and dynamical ac susceptibility, are usually ascribed to the formation of magnetic clusters with no long range magnetic ordering. However, double perovskites showing long-range order 
exhibit similar properties that were explained as arising from vibrations of domain-wall within a pinning potential produced by local anisotropy. ${ }^{30}$ Neutron diffraction is therefore needed to elucidate this point. $\chi_{\text {ac }}$ clearly does not obey a Curie law below $100 \mathrm{~K}$ but the $\chi_{\mathrm{ac}}{ }^{-1} v s \mathrm{~T}$ plot (Fig. 7) was fitted to the CurieWeiss law in the range $150-360 \mathrm{~K}$ yielding a Weiss temperature of $\theta \sim-32 \mathrm{~K}$ and an effective paramagnetic moment of $\rho_{\text {eff }}=5.66 \mu_{\mathrm{B}}$ per formula unit. The negative $\theta$ indicates predominant antiferromagnetic exchange interactions and its value is close to the temperature at which the peak in the ZFC curve of dc magnetization measurements is observed. $\rho_{\text {eff }}$ is smaller than the spin-only value for a high spin $\mathrm{Mn}^{2+}$ ion $\left(5.90 \mu_{\mathrm{B}}\right)$ but it nicely agrees with the expected contribution for non-interacting $\mathrm{Mn}^{3+}-\mathrm{Re}^{5+}$ paramagnetic array (5.65 $\mu_{\mathrm{B}}$ for spin-only contributions). However, an intermediate $\mathrm{Mn}^{2.5+}$ $\mathrm{Re}^{5.5+}$ valence state was deduced from the refined bond lengths summarized in table 3. Assuming that the paramagnetic state could be modelled by an array of $\mathrm{x}\left(\mathrm{Mn}^{2+}-\mathrm{Re}^{6+}\right)$ and $(1-\mathrm{x})$ of $\left(\mathrm{Mn}^{3+}-\mathrm{Re}^{5+}\right)$ ionic couples, the effective paramagnetic moment would be $\left(\rho_{\text {eff }}\right)^{2}=x \cdot\left[\left(\rho_{\mathrm{Mn}}{ }^{2+}\right)^{2}+\left(\rho_{\operatorname{Re}}{ }^{6+}\right)^{2}\right]+(1-\mathrm{x}) \cdot\left[\left(\rho_{\mathrm{Mn}}{ }^{3+}\right)^{2}\right.$ $\left.+\left(\rho_{\operatorname{Re}}^{5+}\right)^{2}\right]$. If we consider spin-only values for the Mn cations and the total angular momentum $\mathrm{J}$ for the Re cations, the experimental effective paramagnetic moment yields a value of $\mathrm{x}=0.51$, which supports the description in terms of intermediate valence states. The use of $J$ values for Re atoms is justified by the fact that circular dichroism measurements have found orbital moments of $0.3 \mu_{\mathrm{B}}$ per Re atom in related double perovskites. ${ }^{31}$ Therefore, significant spin-orbit coupling is found in Re ions and the angular moment is not quenched for this $5 d$-ion in the system studied.

Fig. 8 shows magnetic loops at selected temperatures. Ferromagnetic contribution is observed at $85 \mathrm{~K}$ whereas the sample behaves as a conventional paramagnet at $95 \mathrm{~K}$. Upon cooling, both the spontaneous magnetization and the coercive field increase. The hysteresis cycle at $5 \mathrm{~K}$ does not reach magnetic saturation at $5 \mathrm{~T}$. In order to check it, magnetic loops were measured at $5 \mathrm{~K}$ in a Quantum Design ppms system using magnetic fields up to $9 \mathrm{~T}$ but the saturation is not achieved even at this field as can be seen in the additional information. Absence of magnetic saturation, under an applied field as high as $30 \mathrm{~T}$, was found for several Re-based double perovskites. ${ }^{32-33}$ This property was ascribed to the existence of 
large disorder at the grain boundary regions which are magnetically harder than the grain core. The obtained magnetic moment is $2.22 \mu_{\mathrm{B}} /$ f.u. at $9 \mathrm{~T}$. This value is significantly smaller than the expected one for a ferrimagnetic ordering of $\mathrm{Mn}^{2+} / \mathrm{Re}^{6+}$. However it is larger than the expected value for $\mathrm{Mn}^{3+} / \mathrm{Re}^{5+}$ order, in agreement with the conclusions of intermediate valences for both $\mathrm{Mn}$ and $\mathrm{Re}$ atoms.

The hysteresis loops provides more interesting information. The initial magnetization curves are in agreement with a pinning-type magnet ${ }^{34}$ which may be related to the irreversibility effects observed in the Fig. 6. This is not surprising because this is a ceramic material with grain boundaries and, furthermore, defects such as vacancies or dislocations are likely to occur. The loop at $5 \mathrm{~K}$ exhibits a remanence of $0.79 \mu_{\mathrm{B}} / \mathrm{f}$.u. This remanence decreases with the temperature. The temperature dependence is not linear but it follows a sigmoid-like function (inset A of Fig. 8). The most striking point is the anomalous temperature dependence for the coercive field $\mathrm{H}_{\mathrm{C}}(\mathrm{T})$. The temperature coefficient is negative in the whole temperature range but a strong increase of the coercive field is observed below $25 \mathrm{~K}$ (inset B of Fig. 8). The presence of defects could explain the large coercive fields but it is very unlikely that a sudden change in the pinning forces would occur with decreasing the temperature. Therefore, this anomaly can be attributed to an abrupt increase of the magnetocrystalline anisotropy.

\section{Conclusions}

$\mathrm{Pb}_{2} \mathrm{MnReO}_{6}$ can be prepared by conventional ceramic procedures. It belongs to the family of double perovskites with an ordered arrangement of $\mathrm{Mn}$ and Re atoms in the B-sublattice. The reported sintering at $710^{\circ} \mathrm{C}$ clearly favours a full Mn-Re ordering. We have shown that this compound undergoes a structural phase transition at $410 \mathrm{~K}$ from a cubic $(F m \overline{3} \mathrm{~m})$ to a monoclinic $(C 2 / \mathrm{m})$ cell on cooling. This transition is driven by the condensation of two primary modes: $\Gamma_{4}{ }^{+}$associated to the anti-phase tilting of $\mathrm{BO}_{6}$ octahedra about the unique $\mathrm{b}$-axis and $\Sigma_{2}$ associated to the collinear movement in opposite directions of some atoms, in particular of $2 / 3$ of the $\mathrm{Pb}^{2+}$ cations. To the best of our knowledge, this type of antiferrodistortive structure has not been reported in the field of double perovskites. 
The structural transition is associated with an anomaly in the thermal expansion of the unit cell due to a significant difference between the volumes of the high and low temperature phases. The variation of lattice constants is anisotropic because on warming, the monoclinic $a$-axis shrinks at the transition temperature whereas the $b$-axis expands. The entropy content for the transition is smaller than the one expected for an order-disorder transition indicating the presence of significant disorder in the low temperature phase. The phase transition is also accompanied by anomalies in the electrical resistivity related to an increase of the activation energy upon cooling the sample.

The magnetic measurements reveal the presence of ferrimagnetic interactions at low temperatures. However, the observed irreversibility in the magnetization curves and dynamic behaviour of the ac susceptibility could be as well due to magnetic inhomogeneities with the presence of either spin-glass region or a reentrant transition. Domain wall pinning effects in long range ferrimagnetic systems could also account for these properties. Neutron diffraction experiments are therefore desirable to clarify this subject.

A sharp increase of the coercive field in the hysteresis loops reveals an abrupt change in the magnetocrystalline anisotropy whose origin might be associated to structural changes in the ferrimagnetic phase. The sample does not achieve magnetic saturation at $9 \mathrm{~T}$ and at $5 \mathrm{~K}$ but the paramagnetic region follows a Curie-Weiss law. Both the effective paramagnetic moments and the magnetization at $9 \mathrm{~T}$ do not fit to $\mathrm{Mn}^{2+}$ and $\mathrm{Re}^{6+}$ valences for the B-sublattice. Our result point out to a mixed valence character $\left(\mathrm{Mn}^{2+\delta}\right.$ and $\left.\operatorname{Re}^{6-\delta} ; \delta \sim 0.5\right)$, also supported by the refined bond lengths obtained in the structural study.

Acknowledgments The authors thank to the Servicio de difracción de rayos $\mathrm{X}$ from Zaragoza University for technical assistance and data acquisition. Financial support from the Spanish MICINN (project FIS08-03951) and DGA (Camrads) is acknowledged. V.C. thanks the FPU research grant from MEC.

Supporting Information Available: Details of the refinements using P $2_{1} / \mathrm{n}$ and $\mathrm{I} 2 / \mathrm{m}$ models. Plots of the volume thermal expansion range, of the activated conductivity and of the isothermal magnetization at $5 \mathrm{~K}$ and up to $9 \mathrm{~T}$. 


\section{References}

1. K. Kimura, T. Goto, H. Shintani H., K. Ishizka, T. Arima and Y. Tokura, Nature (London) 2003, 426, 55 .

2. M. Gajek M, M. Bibes M, S. Fusil S, K. Bouzehouane K, J. Fontcuberta J, A. E. Barthelemy AE and A. Fert, Nat. Mater. 2007, 6, 296.

3. W. Eerenstein, N. D.Mathur and J. F. Scott JF, Nature (London) 2006, 442, 759.

4. J. Wang, J. B. Neaton, H. Zheng, V. Nagarajan, S. B. Ogale, B. Liu, D. Viehland, V. Vaithyanathan, D. G. Schlom, U. V. Waghmare, N. A. Spaldin, K. M. Rabe, M. Wuttig and R. Ramesh, Science 2003, 299, 1719.

5. N. A. Hill, J. Phys. Chem. B 2000, 104, 6694.

6. M. Azuma, K. Takata, T. Saito, S. Ishiwata, Y. Shimakawa and M. Takano, J. Am. Chem. Soc. 2005, 127,8889 .

7. G. Blasse, J. Inorg. Nucl.Chem. 1965, 27, 993.

8. J. W. G. Bos and J. P. Attfield, Chem. Mater. 2004, 16, 1822.

9. J. Blasco, C. Ritter, L. Morellon, P. A. Algarabel, J. M. De Teresa, D. Serrate, J. García and M. R. Ibarra, Sol. State Sci. 2002, 4, 651.

10. M. Retuerto, M. García-Hernández, M. J. Martínez-Lope, M. J. Fernández-Díaz, J. P. Attfield and J. A. Alonso, J. Mat. Chem. 2007, 17, 3555.

11. R. Nechache, C. Harnagea, L. P. Carignan, O. Gautreau, L. Pintilie, M. P. Singh, D. Menard, P. Fournier, M. Alexe and A.Pignolet A., J. Appl. Phys. 2002, 35, 233.

12. C. A. Randall, A. S. Bhalla, T. R. Shrou and L. E. Cross, J. Mater. Res. 1990, 5, 829.

13. N. Rivezzi and Ph. Sciau, J. Sol. State Chem. 1998, 139, 332.

14. P. M. Woodward and K. Z. Baba-Kishi, J. Appl. Cryst. 2002, 35, 233.

15. G. Baldinozzi, Ph. Sciau and P. A. Buffat, Solid State Commun. 1993, 86, 541.

16. J. Blasco, R. I. Merino, J. García and M. C. Sánchez, J. Phys.: Condens. Matter 2006, 182261. 
17. S. A. Larrégola, J. A. Alonso, J. C. Pedregosa, M. J. Martínez-Lope, M. Algueró V. De la PeñaO’shea, F. Porcher, F. Illas, Dalton Trans., 2009, 28, 5453.

18. K. Ramesha, L. Sebastian, B. Eichhorn and J. Gopalakrishnan, Chem. Mater. 2003, 15, 668.

19. G. Subías, J. Blasco, J. García, J. Herrero-Martín and M. C. Sánchez, J. Phys.: Condens. Matter 2009, 21, 075903.

20. Ll. Balcells, J. Navarro, M. Bibes, A. Roig. B. Martínez and J. Fontcuberta, Appl. Phys. Lett. 2001, 78, 781 .

21. J. Rodríguez-Carvajal, Physica B 1993, 192, 55. See also http://www.ill.eu/sites/fullprof/

22. R. D. Shannon, Acta Cryst. A 1976, 32, 751.

23. A. Muñoz, J. A. Alonso, M. T. Casais, M. J. Martínez-Lope and M. T. Fernández-Díaz, J. Phys.: Condens. Matter 2002, 14, 8817.

24. A. M. Glazer,. Acta Cryst. A 1975, 31, 756.

25. A. LeBail, H. Duroy and J. L. Bouquet, Mat. Res. Bul. 1988, 23, 447.

26. B. J. Campbell, H. T. Stokes, D. E. Tanner and D. M. Hatch, J. Appl. Cryst. 2006, 39, 607.

27. D. Orobengoa, C. Capillas, M. I. Aroyo and J. M. Pérez-Mato, J. Appl. Cryst. 2009, 42, 820.

28. M. V. Gorev, I. N. Flerov, V. S. Bondarev and Ph. Sciau, Phys. Solid State 2002, 44, 353.

29. M. V. Gorev, I. N. Flerov and Ph. Sciau, Phys. Solid State 2001, 43, 345.

30. J. Blasco, J. García, M. C. Sánchez, J. Campo, G. Subías and J. Pérez-Cacho, Eur. Phys. J. B 2002, 30, 469 .

31. M. Sikora, D. Zajac, M. Borowiec, Cz. Kapusta, C. J. Oates, D. Rybicki, J. M. De Teresa, and M. R. Ibarra, Appl. Phys. Lett. 2006, 89, 062509.

32. J. M. Michalik, J. M. De Teresa, J. Blasco, P. A. Algarabel, M. R. Ibarra, Cz. Kapusta and U. Zeitler, J. Phys.: Condens. Matter 2007, 19, 506206.

33. J. M. De Teresa, J. M. Michalik, J. Blasco, P. A. Algarabel, M. R. Ibarra, Cz. Kapusta and U. Zeitler, Appl. Phys. Lett. 2007, 90, 252514.

34. G. C. Hadjipanayis and A. Kim, J. Appl. Phys. 1988, 63, 3310. 


\section{FIGURE CAPTIONS}

Figure 1. Rietveld refinement of x-ray powder diffraction pattern of $\mathrm{Pb}_{2} \mathrm{MnReO}_{6}$ at $523 \mathrm{~K}$. Observed (points), calculated (line) and the difference below the pattern. The vertical bars correspond to the calculated peak positions for the perovskite $\left(1^{\text {st }}\right.$ row $)$ and pyrochlrore $\left(2^{\text {nd }}\right.$ row $)$ phases. Arrow marks the main peak of the secondary phase. Inset: Picture of the cubic structure for $\mathrm{Pb}_{2} \mathrm{MnReO}_{6}$. Balls denote $\mathrm{Pb}$ atoms while dark (red online) and light (yellow online) octahedra stand for $\mathrm{MnO}_{6}$ and $\mathrm{ReO}_{6}$, respectively.

Figure 2. Rietveld refinement of x-ray powder diffraction pattern of $\mathrm{Pb}_{2} \mathrm{MnReO}_{6}$ at $300 \mathrm{~K}$. Symbols corresponds to the ones of Fig. 1. Inset: Detail of the refinement emphasizing some of the superstructure peaks. The underlined indexes show two very weak peaks not accounted for by the model $C 2 / m$. Asterisk marks the main pyrochlore peak.

Figure 3. Crystal structure of $\mathrm{Pb}_{2} \mathrm{MnReO}_{6}$ projected along the ac-plane at room temperature. Balls stand for $\mathrm{Pb}$ atoms while dark (red online) and light (yellow online) octahedra stand for $\mathrm{MnO}_{6}$ and $\mathrm{ReO}_{6}$, respectively. Arrows indicate the direction for $\mathrm{Pb}$ displacements. The unit cell vectors are also indicated.

Figure 4. Temperature evolution of the lattice parameters for $\mathrm{Pb}_{2} \mathrm{MnReO}_{6}$. The monoclinic $a$ and $c$ axes has been multiplied by the shown factors for the sake of comparison. DSC scans on cooling and heating at $10 \mathrm{~K} \mathrm{~min}^{-1}$.

Figure 5. Electrical resistivity vs. temperature for $\mathrm{Pb}_{2} \mathrm{MnReO}_{6}$. The line and points indicate the measurements under 0 and $1 \mathrm{~T}$, respectively. Inset: Ln $\rho$ vs. 1/T between 300 and $500 \mathrm{~K}$. The lines show the fits to the Arrhenius law above $(500-420 \mathrm{~K})$ and below $(405-300 \mathrm{~K})$ the structural phase transition temperature $(\sim 410 \mathrm{~K})$. The calculated activation energies are also indicated in the figure.

Figure 6. Dc magnetization vs temperature for $\mathrm{Pb}_{2} \mathrm{MnReO}_{6}$. Circles and squares correspond to the measurements under fields of 500 and 5000 Oe. Solid symbols refers to ZFC conditions while open symbols stand for FC conditions. Inset: Ac magnetic susceptibility at frequencies of the alternating field of (from top to bottom) 17, 99 and $999 \mathrm{~Hz}$.

Figure 7. Inverse of the ac magnetic susceptibility vs. temperature for $\mathrm{Pb}_{2} \mathrm{MnReO}_{6}$. The line represents the Curie-Weiss fit obtained between 150 and $360 \mathrm{~K}$. The fit parameters and the effective paramagnetic moment are also shown.

Figure 8. Isothermal magnetization of $\mathrm{Pb}_{2} \mathrm{MnReO}_{6}$ at the temperatures indicated in the plot. The data at 15 and $25 \mathrm{~K}$ have been omitted for the sake of clarity. The insets show the temperature dependence for the remnant magnetization (inset A) and coercivity (inset B) of the hysteresis loops. 


\begin{tabular}{|c|c|c|}
\hline & $523 \mathrm{~K}$ & $300 \mathrm{~K}$ \\
\hline Space group & $F m \overline{3} m$ & $C 2 / m$ \\
\hline a (Á) & $8.1098(2)$ & $18.2309(9)$ \\
\hline b (Á) & - & $8.0359(2)$ \\
\hline c (Å) & - & $5.7072(2)$ \\
\hline$\beta$ (deg) & - & $108.288(2)$ \\
\hline Vol. $\left(\AA^{3}\right)$ & $533.38(2)$ & $793.88(5)$ \\
\hline $\mathbf{Z}$ & 4 & 6 \\
\hline No. reflections & 97 & 858 \\
\hline Refined parameters & 15 & 32 \\
\hline $\operatorname{Rp}(\%)$ & 5.9 & 4.6 \\
\hline $\mathbf{R}_{\mathrm{wp}}(\%)$ & 8.0 & 6.4 \\
\hline $\mathbf{R}_{\text {Bragg }}(\%)$ & 2.1 & 3.7 \\
\hline
\end{tabular}

Table 1. Refined lattice parameters, unit cell volume and refinement data for $\mathrm{Pb}_{2} \mathrm{MnReO}_{6}$. The number of reflections and refined parameters only concerns to the main perovskite phase. The reliability factors are defined in ref. 21. 


\begin{tabular}{|c|c|c|c|c|c|}
\hline atom & site & $\mathbf{x} / \mathbf{a}$ & $\mathbf{y} / \mathbf{b}$ & $\mathbf{z} / \mathbf{c}$ & $\mathbf{B}\left(\AA^{2}\right)$ \\
\hline \multicolumn{6}{|c|}{ Cubic cell at $523 \mathrm{~K}$} \\
\hline $\mathbf{P b}$ & $8 \mathrm{c}$ & $1 / 4$ & $1 / 4$ & $1 / 4$ & $2.65(3)$ \\
\hline Mn & $4 b$ & $1 / 2$ & $1 / 2$ & $1 / 2$ & $0.34(2)$ \\
\hline $\mathbf{R e}$ & $4 a$ & 0 & 0 & 0 & $0.18(2)$ \\
\hline $\mathbf{O}$ & $24 \mathrm{e}$ & $0.2419(6)$ & 0 & 0 & $2.62(15)$ \\
\hline \multicolumn{6}{|c|}{ Monoclinic cell at $300 \mathrm{~K}$} \\
\hline Pb1 & $4 \mathrm{~h}$ & 0 & $0.7388(4)$ & $1 / 2$ & $1.62(14)$ \\
\hline $\mathbf{P b 2}$ & $8 \mathrm{j}$ & 0.8333 & $0.2502(3)$ & $0.8022(2)$ & $0.76(5)$ \\
\hline Mn1 & $2 \mathrm{a}$ & 0 & 0 & 0 & $0.21(7)$ \\
\hline Mn2 & $4 i$ & 0.8333 & $1 / 2$ & $0.3131(11)$ & $0.21(7)$ \\
\hline $\operatorname{Re} 1$ & $2 b$ & 0 & $1 / 2$ & 0 & $0.09(2)$ \\
\hline $\operatorname{Re} 2$ & $4 \mathrm{i}$ & 0.8333 & 0 & 0.3333 & $0.09(2)$ \\
\hline 01 & $4 g$ & 0 & $0.7417(5)$ & 0 & $0.79(16)$ \\
\hline $\mathbf{O 2}$ & $4 i$ & $0.7421(9)$ & $1 / 4$ & $0.0324(18)$ & $0.79(16)$ \\
\hline $\mathbf{O 3}$ & $4 i$ & $0.2265(9)$ & $1 / 4$ & $0.422(3)$ & $0.79(16)$ \\
\hline 04 & $8 \mathrm{j}$ & 0.8333 & $0.2417(5)$ & $0.345(2)$ & $0.79(16)$ \\
\hline 05 & $4 \mathrm{i}$ & $0.5890(10)$ & $1 / 2$ & $0.339(2)$ & $0.79(16)$ \\
\hline O6 & $4 i$ & $0.0912(9)$ & $1 / 2$ & $0.3010(18)$ & $0.79(16)$ \\
\hline $\mathbf{0 7}$ & $4 i$ & $0.0598(9)$ & $1 / 2$ & $0.756(3)$ & $0.79(16)$ \\
\hline 08 & $4 \mathrm{i}$ & $0.5933(10)$ & $1 / 2$ & $0.857(4)$ & $0.79(16)$ \\
\hline
\end{tabular}

Table 2. Wyckoff positions, refined fractional atomic coordinates and isotropic temperature factors for $\mathrm{Pb}_{2} \mathrm{MnReO}_{6}$. An only temperature factor was refined for all oxygen atoms in the monoclinic cell. 


\begin{tabular}{|c|c|c|c|c|c|}
\hline \multicolumn{2}{|c|}{ Cubic at $523 \mathrm{~K}$} & \multicolumn{4}{|c|}{ Monoclinic at $300 \mathrm{~K}$} \\
\hline $\mathrm{Pb}-\mathrm{O}$ & $2.868(4)$ & Pb1-O1 $(\times 2)$ & 2.8537 & Pb2-O1 & $2.8911(1)$ \\
\hline \multirow[t]{8}{*}{$(\times 12)$} & & Pb1-O4(× 2) & $2.890(4)$ & $\mathrm{Pb2-O2}$ & $2.762(11)$ \\
\hline & & Pb1-O5 $(\times 2)$ & $2.970(13)$ & $\mathrm{Pb} 2-03$ & $2.448(8)$ \\
\hline & & Pb1-O6 $(\times 2)$ & $2.987(13)$ & $\mathrm{Pb} 2-\mathrm{O3}$ & $2.610(11)$ \\
\hline & & $\mathbf{P b 1 - O 7}(\times 2)$ & $2.451(9)$ & Pb2-O4 & $3.098(11)$ \\
\hline & & $\mathbf{P b 1 - O 8}(\times 2)$ & $3.045(13)$ & Pb2-O4 & $2.8879(5)$ \\
\hline & & & & $\mathrm{Pb} 2-\mathrm{O5}$ & $2.723(12)$ \\
\hline & & & & Pb2-O6 & $2.603(13)$ \\
\hline & & & & Pb2-08 & $2.825(13)$ \\
\hline$<\mathrm{Pb}-\mathrm{O}>$ & 2.868 & $<$ Pb1-O $>$ & $2.866(3)$ & $<\mathrm{Pb2}-\mathrm{O}>$ & $2.761(3)$ \\
\hline Re-O & $1.962(3)$ & $\operatorname{Re1-O1}(\times 2)$ & $1.942(4)$ & Re2-O2 & $1.981(11)$ \\
\hline \multirow[t]{4}{*}{$(\times 6)$} & & $\operatorname{Re1-O6}(\times 2)$ & $1.981(11)$ & Re2-O3 & $2.024(19)$ \\
\hline & & $\operatorname{Re1-O7}(\times 2)$ & $2.020(19)$ & $\operatorname{Re} 2-04(\times 2)$ & $1.943(4)$ \\
\hline & & & & Re2-O5 & $1.960(11)$ \\
\hline & & & & Re2-O8 & $1.970(23)$ \\
\hline$<\operatorname{Re}-\mathrm{O}>$ & 1.962 & $<\operatorname{Re1-O}>$ & $1.981(5)$ & $<\operatorname{Re} 2-\mathrm{O}>$ & $1.970(6)$ \\
\hline Mn-O & $2.093(3)$ & Mn1-O1 $(\times 2)$ & $2.076(4)$ & Mn2-O2 & $2.019(11)$ \\
\hline \multirow[t]{4}{*}{$(\times 6)$} & & $\operatorname{Mn1-O5}(\times 2)$ & $2.099(11)$ & Mn2-O3 & $2.222(18)$ \\
\hline & & Mn1-O8 $(\times 2)$ & $2.105(22)$ & Mn2-O4( $\times 2)$ & $2.084(4)$ \\
\hline & & & & Mn2-O5 & $2.200(11)$ \\
\hline & & & & Mn2-O8 & $2.106(18)$ \\
\hline$<\mathrm{Mn}-\mathrm{O}>$ & 2.093 & $<$ Mn1-O $>$ & $2.093(6)$ & $<$ Mn2-O $>$ & $2.119(5)$ \\
\hline
\end{tabular}

Table 3. Selected refined bond distances ( $⿱$ Á) for $\mathrm{Pb}_{2} \mathrm{MnReO}_{6}$. 


\begin{tabular}{|c|c|c|}
\hline Space group & Lattice vectors & Origin \\
\hline$C 2 / m($ No. 12$)$ & $(-1 / 2,-1 / 2,0),(0,0,1),(-1,2,0)$ & $(0,0,0)$ \\
\hline$P 2 / c($ No. 13$)$ & $(-1 / 2,-1 / 2,0),(0,0,1),(-1,2,0)$ & $(0,0,0)$ \\
\hline$P 2_{1} / c($ No. 14$)$ & $(-1 / 2,-1 / 2,0),(0,0,1),(-1,2,0)$ & $(0,0,0)$ \\
\hline \multicolumn{3}{|c|}{ Refined global amplitudes of representations } \\
\hline$\Gamma_{1}^{+}$ & 0.11 & \\
\hline$\Gamma_{4}^{+}$ & 0.9 & \\
\hline$\Gamma_{5}^{+}$ & 0.4 & \\
\hline$\Sigma_{1}$ & 0.10 & \\
\hline$\Sigma_{2}$ & 0.6 & \\
\hline
\end{tabular}

Table 4. Space group, lattice vectors and origin respect to the parent cell (high temperature phase). The cells retained have $2 / \mathrm{m}$ point group and $\Sigma_{2}$ is the driving mode to produce the observed superstructure peaks. Refined global amplitudes of irreducible representations in the refinement with the $C 2 / \mathrm{m}$ cell. 
Figure 1.

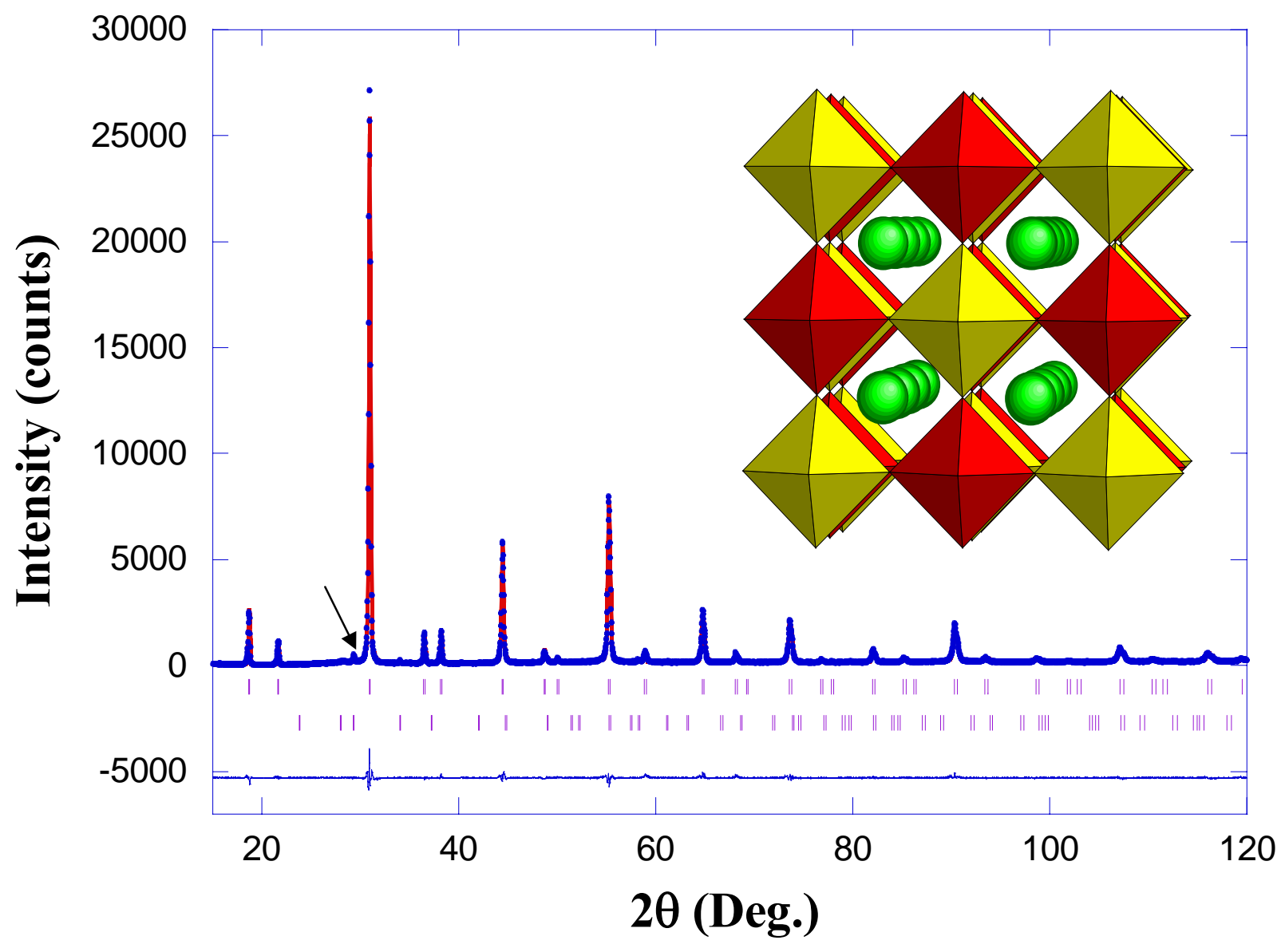


Figure 2.

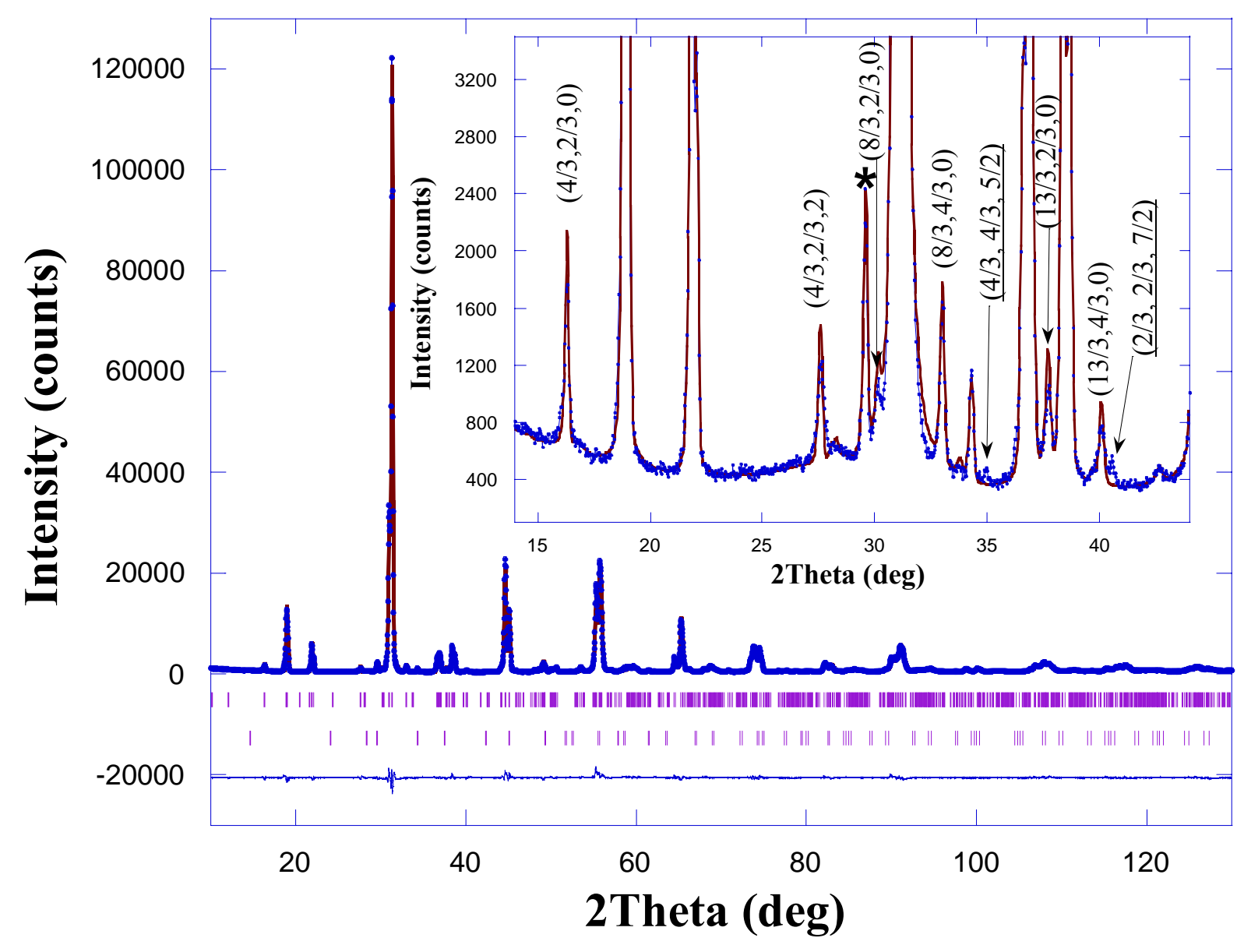


Figure 3.

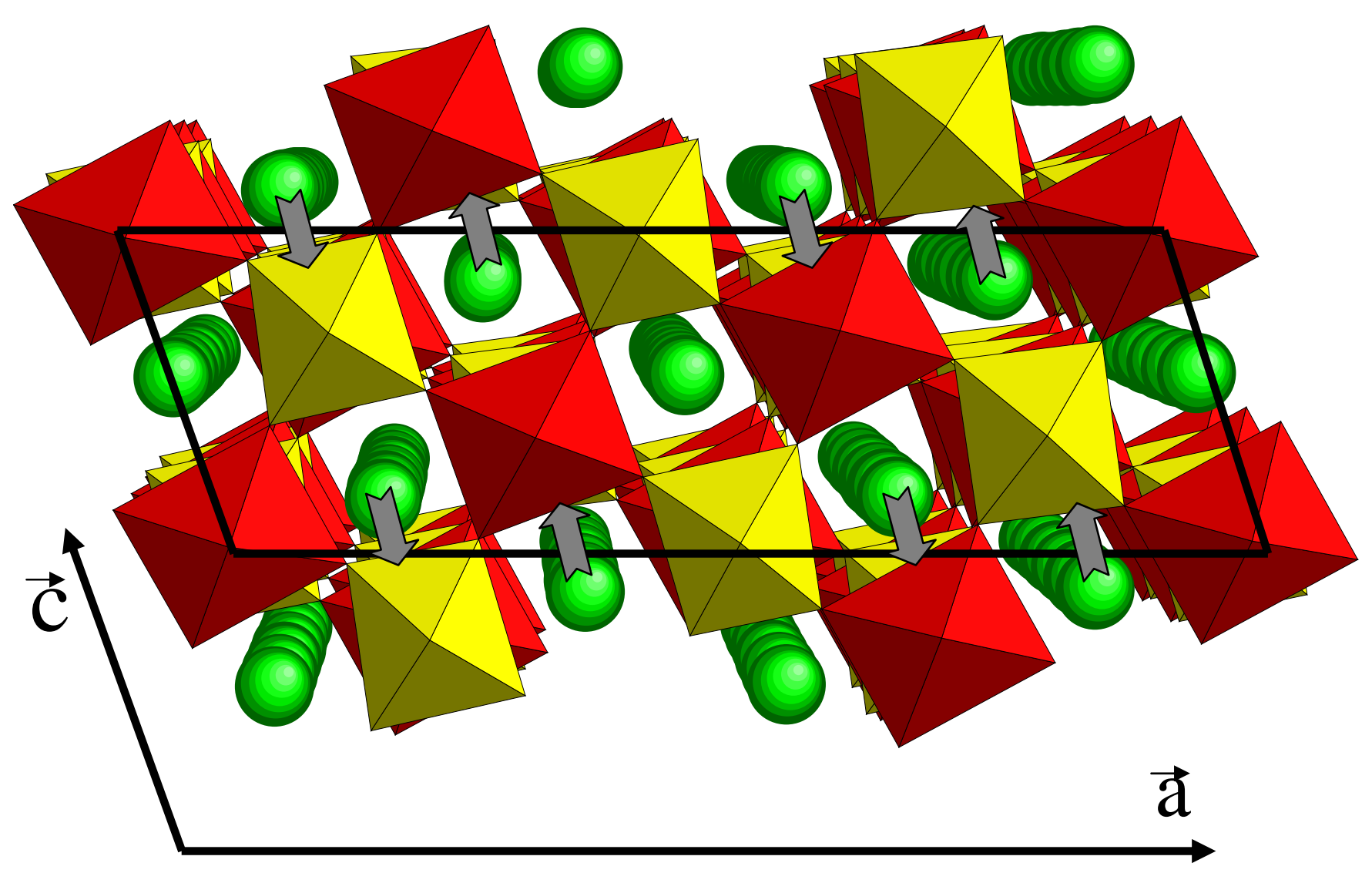


Figure 4.

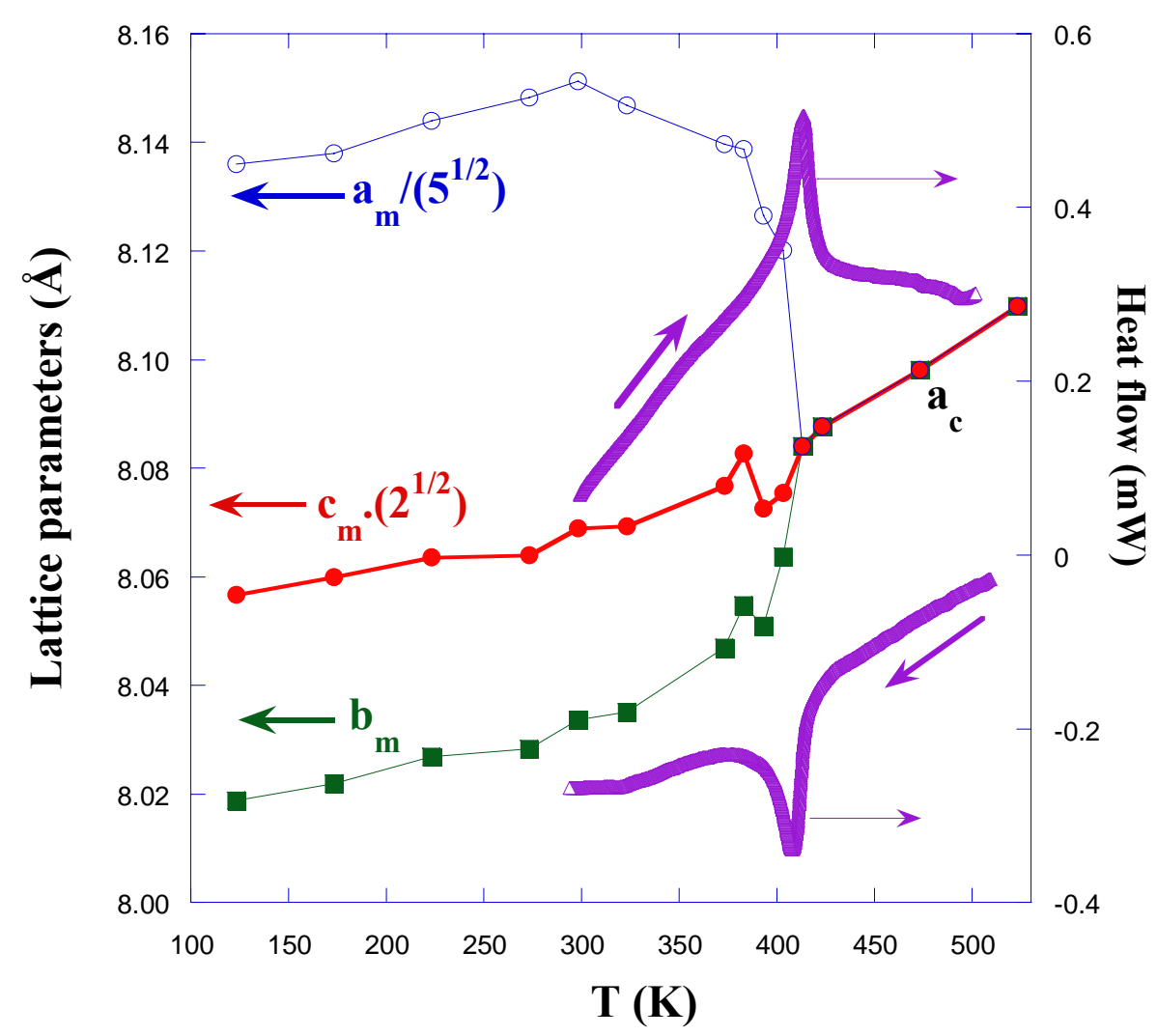


Figure 5.

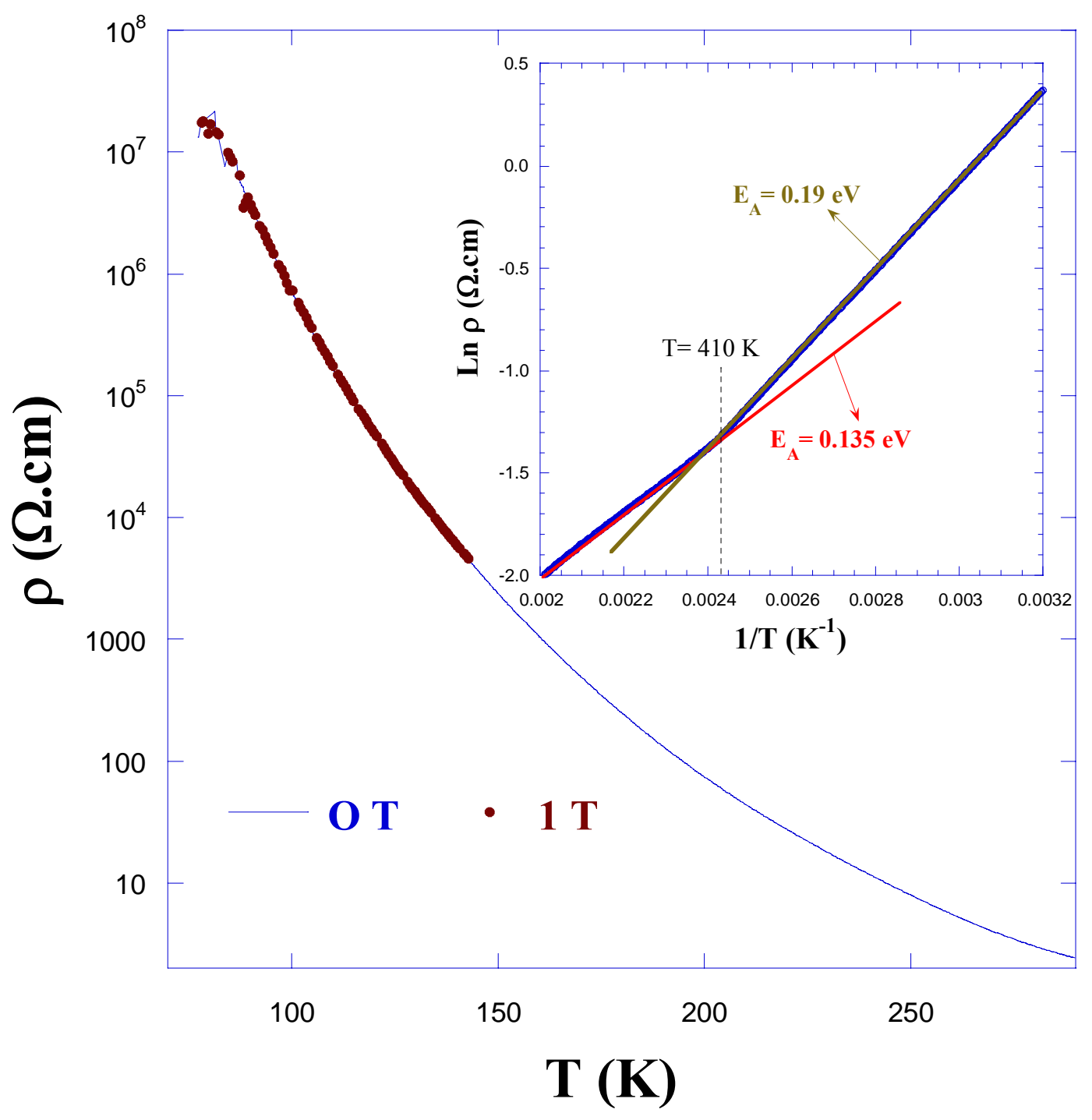


Figure 6.

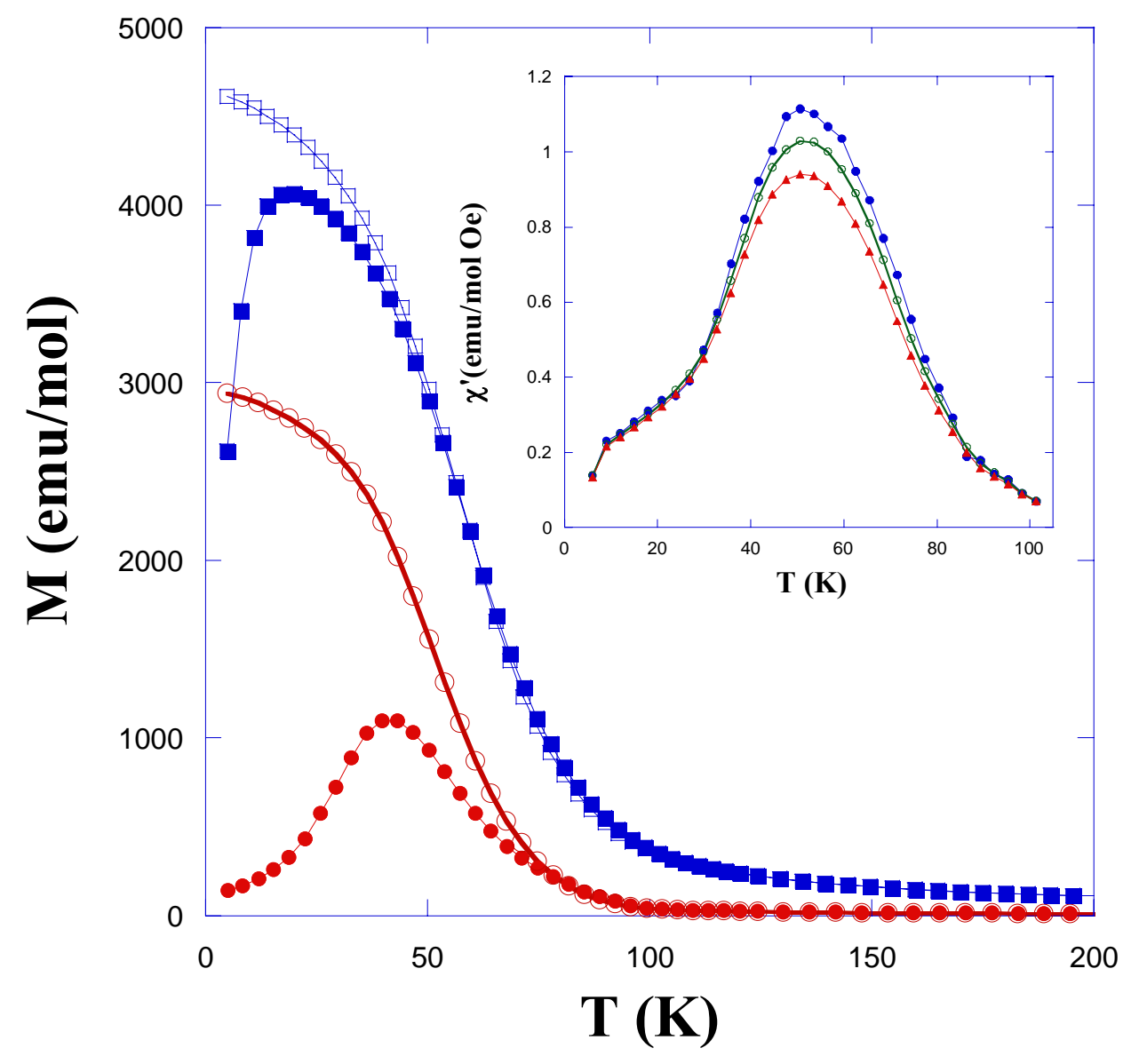


Figure 7.

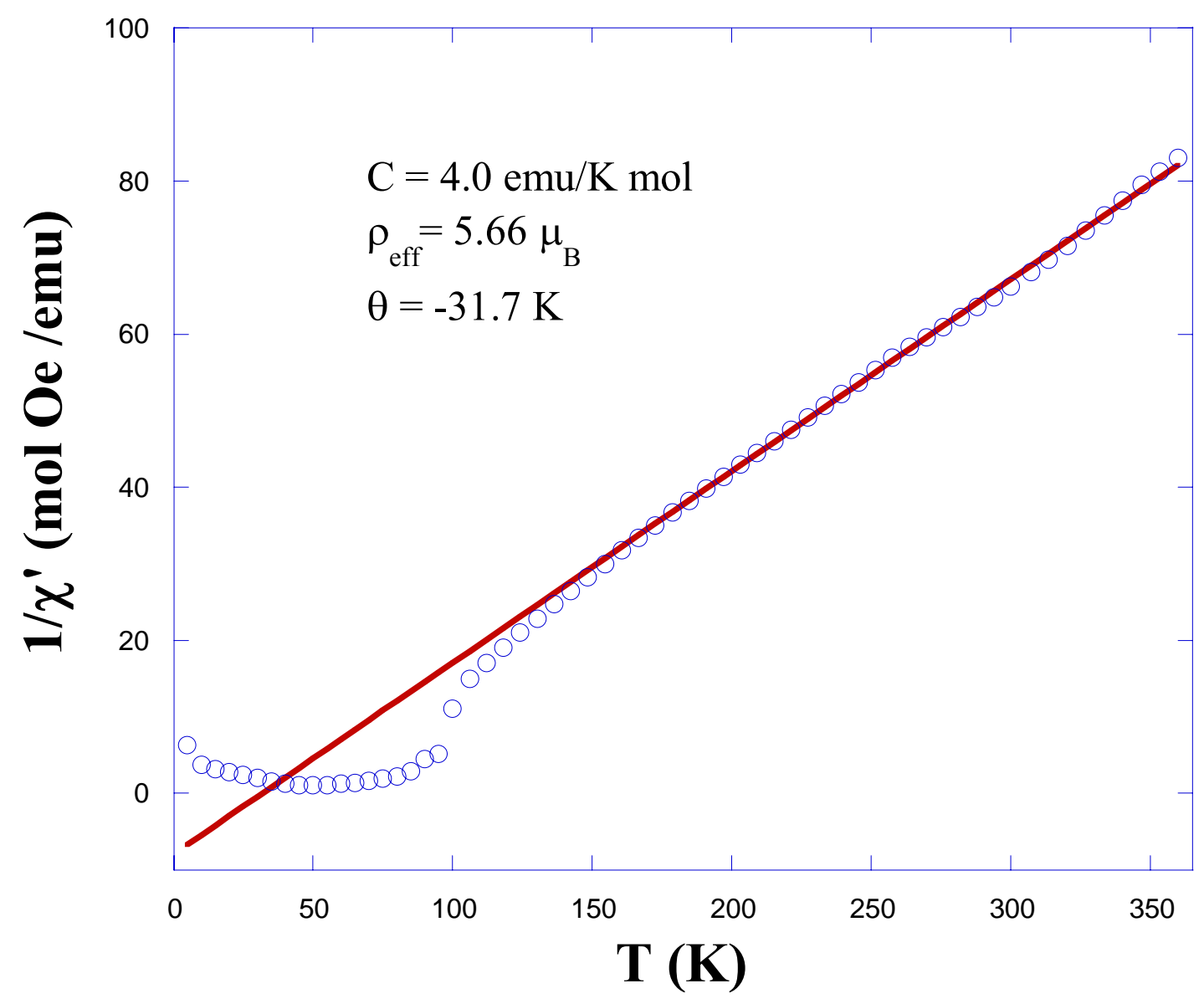


Figure 8.

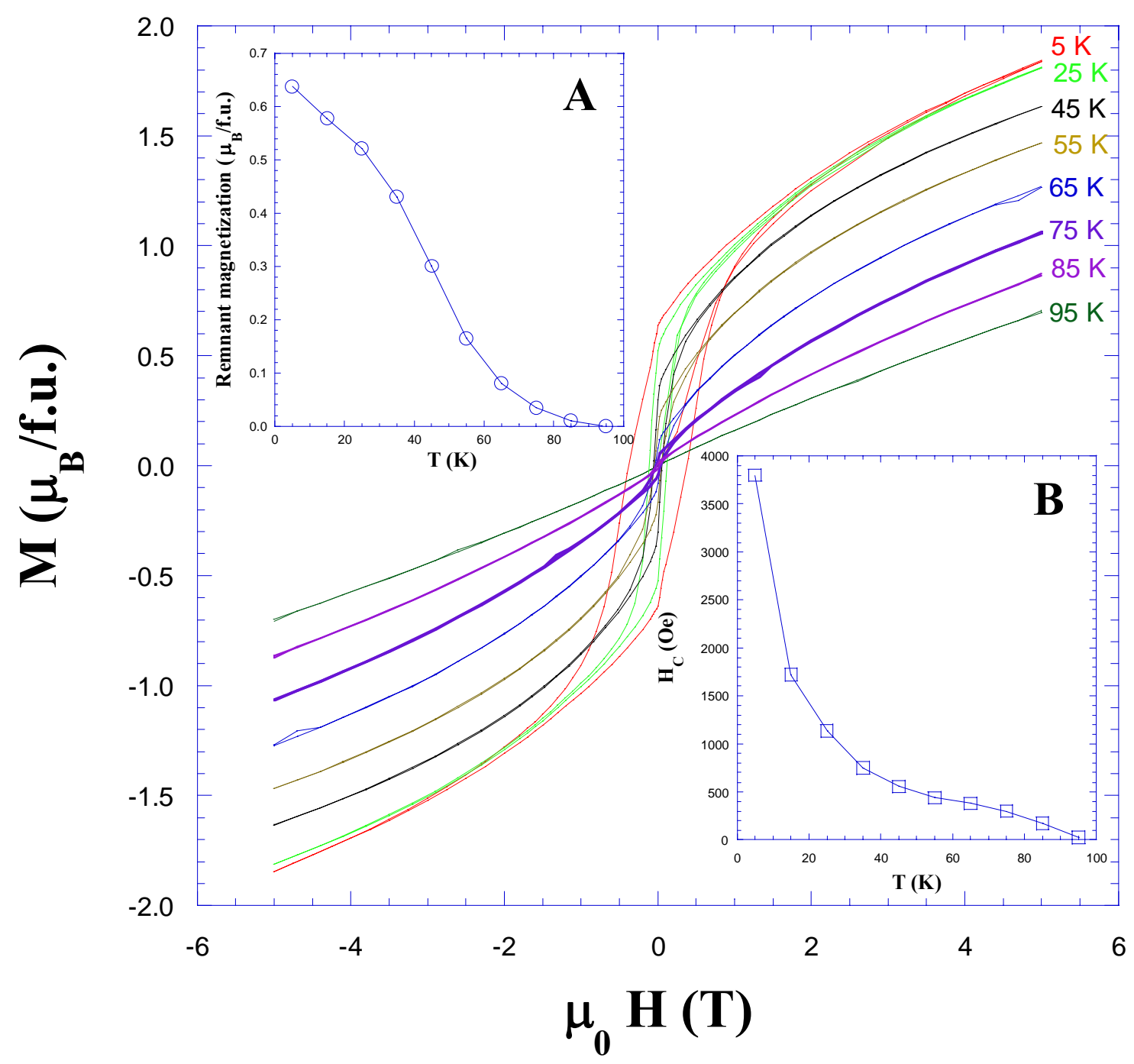

